\title{
Parametric design study of a substrate material for a DEMO sacrificial limiter
}

\author{
R. de Luca*a, P. Fanellia, S. Mingozzi ${ }^{b}$, G. Calabròa, F. Vivio ${ }^{b}$, F. Mavigliac and J. H. You ${ }^{d}$ \\ ${ }^{a}$ University of Tuscia, DEIm Department, Via del Paradiso 47, 01100 Viterbo, Italy \\ ${ }^{b}$ University di Rome "Tor Vergata”, Enterprise Engineering Department, Via del Politecnico 1, 00133, Rome, Italy \\ ${ }^{c}$ EUROfusion Consortium, PPPT Department, Garching, Boltzmannstr. 2, Germany \\ ${ }^{d}$ Max Planck Institute for Plasma Physics, Boltzmann Str. 2, 85748 Garching, Germany
}

\begin{abstract}
Towards the realization of nuclear fusion, a future reactor must provide efficient and safe power exhaust through both the divertor and FW. Recent studies suggest that the greatest engineering challenges of plasma-facing components (PFCs) may arise from the occurrence of plasma transients, when extreme heat fluxes are expected. In severe cases, extensive surface vaporization, melting and re-solidification may lead to excessive degradation of conventional PFCs. An eventual failure would compromise the reactor safety as well as its prompt return to normal operation. A sacrificial limiter provided with a micro-engineered substrate material is being investigated for DEMO to cope with the harsh conditions occurring during unmitigated plasma disruptions. Among the possible solutions, innovative materials such as tungsten $(\mathrm{W})$ based lattice structures can be tailored to meet functional requirements and prevent severe failures. As a further step in this direction, an equivalent solid model, originally developed and validated for open-cell $\mathrm{Al}$ foams, was transferred to $\mathrm{W}$ foams and the scaling law of its thermo-physical properties evaluated as a function of the most influential parameters. In the present work, a design optimization tool for the thermal behavior of the substrate material is presented. The latter is modeled as a homogeneous material having equivalent properties. A parametric design study was carried out to assess their impact on the global behavior of the PFC. Independent combinations of equivalent thermal conductivity and density have been applied to the substrate material by scaling the corresponding properties of bulk W. For each design point, results were tabulated and compared with user-defined operating requirements. Afterwards, a post-processing routine was implemented for effective visualization of the space of solutions. Ultimately, tailored lattice structures potentially able to fulfill the operating requirements are proposed. An existing solid model for open-cell $\mathrm{W}$ foams was employed to design the features of such material based on the results of the parametric study.
\end{abstract}

Keywords: DEMO, first wall, limiter, plasma-facing component, lattice structures, topology optimization, FEA

\section{Introduction}

Controlling the heat and particle exhaust inside a tokamak is a key issue towards the realization of nuclear fusion [1]. Inside a future tokamak, PFCs must withstand harsh operating conditions such as high heat flux, erosion and sputtering as well as additional limitations owing to the presence of a consistent neutron flux [2]. In state-ofthe-art plasma-facing components (PFCs), W has been preferred as armor material owing to its high melting point, acceptable thermal conductivity, high sputtering threshold and hydrogen compatibility. On the other hand, it is brittle at room temperature. Moreover, a thermal expansion mismatch exists between $\mathrm{W}$ and chosen heat sink materials. Considering DEMO and future reactors, recent studies suggest that the greatest challenges of PFCs may arise from the occurrence of plasma transients, such as plasma limited phases, vertical displacement events (VDE) and disruptions [3]. Assuming a plasma thermal energy content of $1.3 \mathrm{GJ}$ and a scrape-off layer (SOL) broadening factor of 7, in accordance with [4], a DEMO unmitigated disruption thermal quench (TQ) was simulated. The estimated wall power density was in the order of tens of $\mathrm{GW} / \mathrm{m}^{2}$ for the $4 \mathrm{~ms}$ long deposition time assumed [3]. This suggests that no existing materials would be able to survive such an extreme amount of energy deposition, largely above the melting limit for W, which is around $50 \mathrm{MJ} / \mathrm{s}^{0.5} \mathrm{~m}^{2}$ [5]. Surface vaporization, melting and re-solidification is therefore expected. In severe cases, eventual failure of conventional PFCs might compromise the reactor safety and delays its return to normal operation. Therefore, wall protection strategies are being considered to prevent the first wall (FW) from excessive degradation. Among them, we can mention pellet injection and gas puffing. In this regard, TOKES code simulations carried out by $\mathrm{S}$. Pestchanyi et al. provided encouraging results, with the wall power density during such transients significantly reduced owing to vapor shielding [6].

Combined with mitigation and prevention strategies, first wall limiters seem to be unavoidable in DEMO to prevent the unshadowed first wall from plasma transients, as stated in [7]. In this context, we aim to investigate a sacrificial PFC to cope with unmitigated plasma disruptions in DEMO. Such component is provided with newly-engineered materials tailored to fulfil its functional (and conflicting) requirements. In fact, during normal operation, heat exhaust capability must be guaranteed to exhaust the steady-state load (anticipated to be around $0.5-1 \mathrm{MW} / \mathrm{m}^{2}$ in DEMO [3]). This requires an actively cooled heat sink and adequate thermal conductivity. On 
the other hand, when the disruption occurs, the vapor shielding formation must be promoted to reduce (and lock) the impinging heat flux. At the same time, overloading and failure of the heat sink must be avoided. This can be only achieved with a perfect thermal insulation of the heat sink from the sacrificial armor, which is a requirement conflicting with the steady-state requirement. In addition, the possibility to survive a large number of transient events before the replacement of the sacrificial limiter is also desired. Micro-engineered structures can be designed to fulfil these challenging requirements. Innovative materials might act as a thermal "air-bag" during thermal shock while providing acceptable performances during normal operations. Although targeting inertial fusion energy applications, Wbased micro-engineered armors, such as micro-machined structures, plasma sprayed coatings, lattice structures and foams have been investigated in [8]. Among them, metal foams and lattice structures turned out to be promising candidates for sacrificial PFC applications, having low thermal stresses at high temperatures and moderate thermal diffusivity. Unlike bulk W, these properties could promote a prompt vapor shielding formation, which is expected to decrease significantly (and lock) the impinging heat flux during disruptive events up to 8-10 times [6]. In literature, design optimization techniques for divertor and first wall applications comprises topology optimization [9] and fabrication of functionally graded materials [10]. These can help mitigate the thermal expansion mismatch between armor and heat sink material. In this perspective, thermo-physical characteristics of lattice structures can be calibrated with a proper choice of ligament and cell features, i.e. the ligament length, ligament cross section, pore density and cell anisotropy (i.e. the aspect ratio of the unit cell). As the first step in this direction, an existing solid model originally developed by P. Fanelli et al. [11] for Al-based open-cell foams was transferred to $\mathrm{W}$ foams [12]. The impact of the most influential foam parameters on thermal conductivity and density was investigated. The parametric approach has dual advantages: such a model can either be tuned on available measurements for FE analyses or be used for design optimization. However, further studies are yet to be done to identify the proper set of material features for a sacrificial PFC.

In this work, we present a FE-based tool developed for parametric design optimization of a substrate material to be used in a DEMO FW sacrificial limiter. The influence of substrate thickness and smeared thermo-physical properties on the global response of the component have been assessed. Based on these results, tailored lattice structures have been proposed able to fulfil user-defined operating criteria.

\section{Model and Materials}

The final design of the limiter was unknown at this early stage, so a flat tile design with a circular cooling channel was at first assumed as a baseline. The component width and the circular cooling channel were set according to the current DEMO monoblock design [13]. Eventual design changes that might occur can be easily implemented in the present model. A schematic configuration of the PFC is reported in Fig.1. The model comprises three main parts: the armor, the substrate material and the heat sink. The armor material is the plasma-facing material. It consists of a bulk W layer employed to increase the lifetime of the limiter thus the number of transients that can be faced before its replacement. Existing studies on the W monoblock design predict a few hundred microns of melt $\mathrm{W}$ after a single disruption [14]. Therefore, a few mm of dense $\mathrm{W}$ armor should be enough for an acceptable component lifetime. Nonetheless, a further option under study consists of facing directly the plasma with the substrate material. This latter is a porous $\mathrm{W}$ structure having micro-engineered features that provide it with peculiar performances. In the present layout, these geometrical features are neglected. Nonetheless, the substrate was modelled as a homogeneous material having smeared thermo-physical properties that depend on these features. For this purpose, a scaling factor $f$, ranging from 0.1 to 1 , was applied to the density and thermal conductivity of bulk $\mathrm{W}$, both available in literature as functions of the operating temperature [15]:

$$
\begin{aligned}
& \rho(T)=1000 \cdot\left(19.3027-2.3786 \mathrm{e}-4 T-2.2448 \mathrm{e}-8 T^{2}\right) \\
& \lambda(T)=174.9274-0.1067 T+5.0067 \mathrm{e}-5 T^{2}-7.8349 \mathrm{e}-9 T^{3}
\end{aligned}
$$

As a simplifying and conservative assumption, $\mathrm{W}$ melting and vaporization were not included in this analysis. Nonetheless, studies are ongoing to implement more detailed FE analyses with $\mathrm{W}$ melting, vaporization and vapor shielding. The influence of the operating temperature was maintained. The scaling factors applied to both density and thermal conductivity are taken as optimization parameters. Therefore, independent combinations of these properties can be applied to the substrate material. The average specific heat $\bar{c}_{p}$ is reported hereafter as a weighted average depending on $\mathrm{W}$ and a void fraction (i.e. closed porosity, filled with air or inert gas)

$$
\bar{c}_{p}=\frac{1}{1+\frac{\rho_{a} V_{a}}{\rho_{w} V_{w}}}\left(c_{p, w}+c_{p, a} \frac{\rho_{a} V_{a}}{\rho_{w} V_{w}}\right)
$$

where $\mathrm{V}$ is volume and $\rho$ is density. Depending on the manufacturing process, it can be assumed that closed pores are filled with air or inert gas, thus pedix "w" and "a" were used to refer to $\mathrm{W}$ or air. It can be observed that the ratio between their density is in the order of $10^{-5}$, while

$$
\frac{V_{a}}{V_{w}}=\frac{V_{a}}{V_{w}+V_{a}} \frac{V_{w}+V_{a}}{V_{w}}=\frac{p}{1-p}
$$

Therefore, even pronounced values of porosity ( $p$ ) do not affect the specific heat of $\mathrm{W}$. The third part is the heat sink, devoted to exhausting the heat coming from the plasma. In our study, CuCrZr was preferred to steel as it is the current reference heat sink material in state-of-theart targets [13]. It also offers enhanced power exhaust capabilities. Material properties of the above mentioned materials were taken from the ITER SDC-IC [15]. Concerning the armor and substrate thicknesses, four layout variants have been considered, as listed in Table 1. 


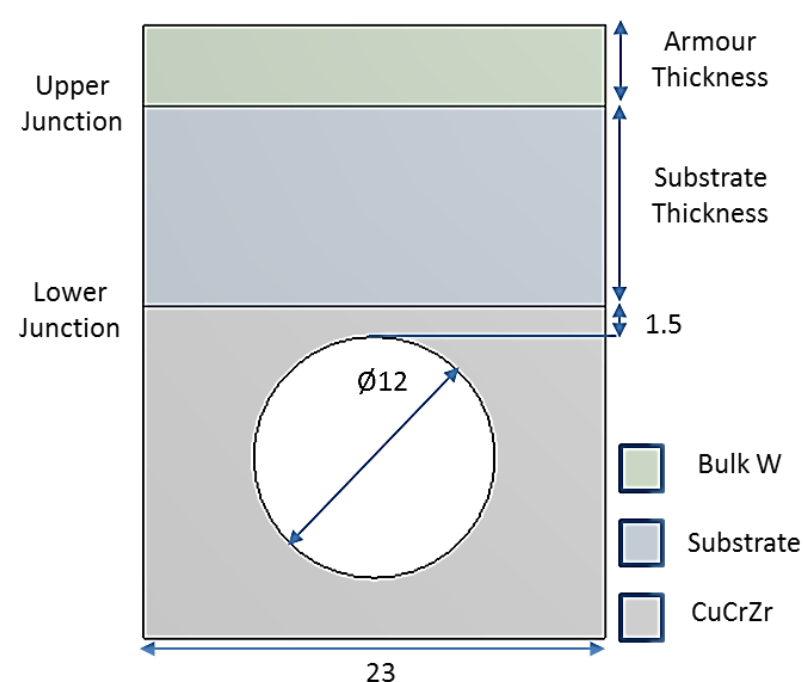

Fig. 1. Schematic layout of the sacrificial PFC

Table 1. Layout variants

\begin{tabular}{ccc}
\hline $\begin{array}{c}\text { Layout } \\
\text { Variant }\end{array}$ & $\begin{array}{c}\text { Armour Thickness } \\
(\mathrm{mm})\end{array}$ & $\begin{array}{c}\text { Substrate Thickness } \\
(\mathrm{mm})\end{array}$ \\
\hline 1 & 0 & 14 \\
2 & 4 & 10 \\
3 & 6 & 8 \\
4 & 10 & 4 \\
\hline
\end{tabular}

Table 2. Water cooling parameters

\begin{tabular}{lc}
\hline Parameter $($ Unit $)$ & Value \\
\hline Pressure $(\mathrm{MPa})$ & 5 \\
Temperature $\left({ }^{\circ} \mathrm{C}\right)$ & 150 \\
Velocity $(\mathrm{m} / \mathrm{s})$ & 16 \\
Pipe diameter $(\mathrm{mm})$ & 12 \\
Swirl tape twist ratio & 2 \\
\hline
\end{tabular}

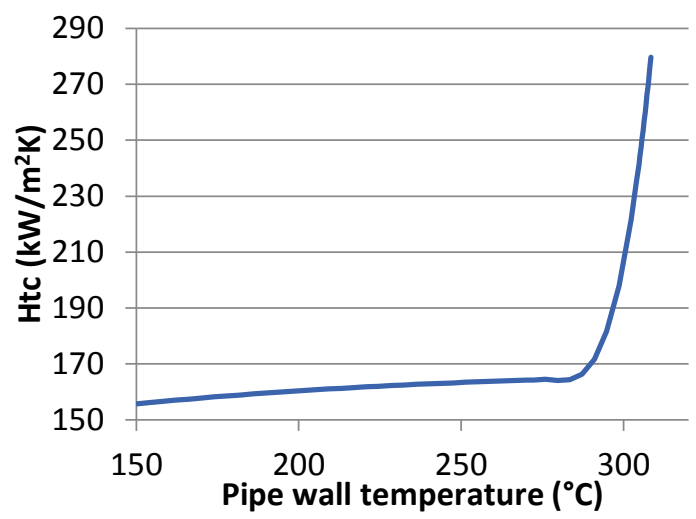

Fig. 2. Htc as a function of the pipe wall temperature

\section{Boundary Conditions}

The above described schematic layout was modelled in ANSYS 18.2 using 2380 PLANE77 8-node thermal elements, with 1 dof per node (temperature) and plane behavior. Only slightly different results were found using finer meshes. In the thermal calculation, the thermal contact resistance was neglected by assuming perfectly bonded interfaces. The disruption TQ was modelled following a steady-state operation under $1.5 \mathrm{MW} / \mathrm{m}^{2}$, which is a quite conservative value compared to the loads expected for the DEMO FW during normal operations [3]. The transient heat flux was defined as a step function. Duration and heat flux magnitude were selected as optimization parameters. The heat pulse lasts from 1 to 10 $\mathrm{ms}$, and its magnitude varies from 1 to $10 \mathrm{GW} / \mathrm{m}^{2}$. Therefore, the energy deposited lays within 1-100 MJ $/ \mathrm{m}^{2}$. The PFC is actively cooled by water in forced convection. Thermo-hydraulic conditions were assumed in accordance with those of the DEMO divertor target [13]. For circular channels, the heat transfer coefficient (htc) can be calculated as a function of the temperature at the pipe wall using SIEDER-TATE-CEA-THOM semiempirical laws [16]. The resulting htc trend is shown in Fig.2. After each heat pulse, free cooling up to $10 \mathrm{~s}$ was modelled to appreciate the temperature increase associated with the heat diffusing to the heat sink.

An additional key parameter for water-cooled PFCs is the critical heat flux (CHF), i.e. the maximum heat flux that can be absorbed by cooling water before reaching the boiling crisis. When the saturation conditions are reached at the pipe wall, nucleate boiling starts with the formation of insulated vapor bubbles. As latent heat removes heat at a constant temperature, htc increases (see Fig.2). Moreover, the flowing water removes the bubbles from the loaded area, preventing the formation of vapor clusters. However, if excessive heat is provided, massive vaporization may lead to the progressive formation of vapor slugs, columns and ultimately stable layers interposed between the pipe wall and water. Therefore, an abrupt reduction in htc occurs leading to wall overheating potentially exceeding material limit. The CHF correlation proposed in [17] was used to predict the occurrence of the boiling crisis. One may correctly point out that the thermal shock duration is expected to affect only the top of the PFC. On the other hand, after the pulse, the energy deposited diffuses towards the pipe. If the thermal diffusivity is high enough, the pipe may locally overcome the CHF limit. Even if this might not be a showstopper due to the limited VDE duration, as also suggested in [7], in absence of experimental evidence it was decided to keep the maximum temperature reached by the pipe wall among the user-defined operating requirements. The model won't lose its validity by changing this limit, because the results filtering is a post-process operation that can be easily customized.

\section{Design Optimization Tool}

The total amount of design points generated for the parametric analysis is equal to 40000 , i.e. 100 independent combinations of substrate density and thermal conductivity, 4 layout variants and 100 independent combinations of thermal shock duration and magnitude. The thermal analysis procedure has been totally automated using an ANSYS routine that sets up and updates the FE models, runs the calculations and, as an outcome, exports data for post-processing. For each design point after both steady-state and transient analysis, 
a number of (user-defined) key parameters were tabulated. Among them, the maximum temperatures reached at the plasma-facing surface, at the substrate's upper junction and lower junction (respectively UJ and LJ) as well as the temperature at the pipe wall. Furthermore, the maximum total heat flux at the pipe wall was tabulated in $\mathrm{W} / \mathrm{m}^{2}$. A post-processing routine was implemented in Matlab to filter and compare data with the following user-defined criteria:

$$
\begin{aligned}
& T_{U J} \leq T_{\text {lim } 1}=3400^{\circ} \mathrm{C} \\
& T_{L J} \leq T_{\text {lim } 2}=400^{\circ} \mathrm{C} \\
& T_{\text {pipe }} \leq T_{\text {lim } 3}=300^{\circ} \mathrm{C}
\end{aligned}
$$

The first limit is the melting temperature of $\mathrm{W}$ to appreciate excessive armor degradation after a single thermal shock. However, as we neglected melting and vaporization at this preliminary stage, the temperatures at the plasma-facing surface may be overestimated, leading to misleading conclusions. In future analysis, this limit could be set to the junction limit that depends on the joining technology adopted for the UJ. The second limit was imposed to account for the worsened thermomechanical properties of the heat sink material due to the significant neutron flux expected on the first wall of DEMO. In the absence of more reliable information from neutronic evidence, the temperature at which over-aging and loss of strength becomes excessive for copper alloy up to 5 dpa was used [2], although if this limit could be reached for a very short time and might not be regarded as a critical showstopper. The third limit was imposed by the choice of a sufficient margin from the CHF.

3D graphs were generated for effective visualization of the space of solutions, i.e. the operating range collecting the design points able to satisfy the imposed operating criteria (Fig. 3 and 5). Design points are marked with a green circle when all the criteria are satisfied. Instead, red marks appear when none of them is verified. When the UJ condition is not satisfied, the design point is marked with a blue triangle whereas a pink one appears when the LJ condition is not verified. Ultimately, a square mark is applied when the CHF limit is reached.

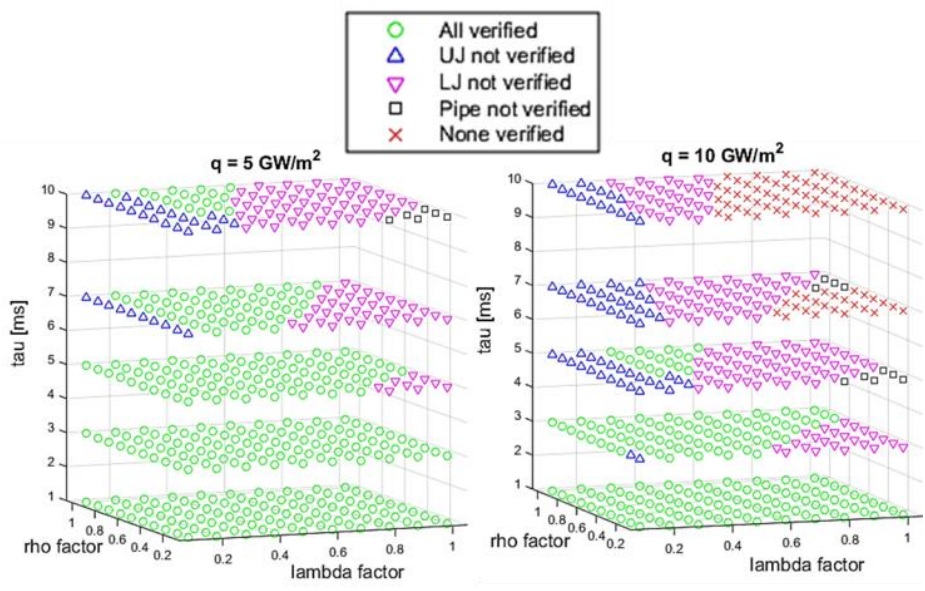

Fig. 3. Impact of the substrate material and pulse duration for layout variant 2 , (fully dense $\mathrm{W}$ corresponds to rho and lambda factors equal to 1)

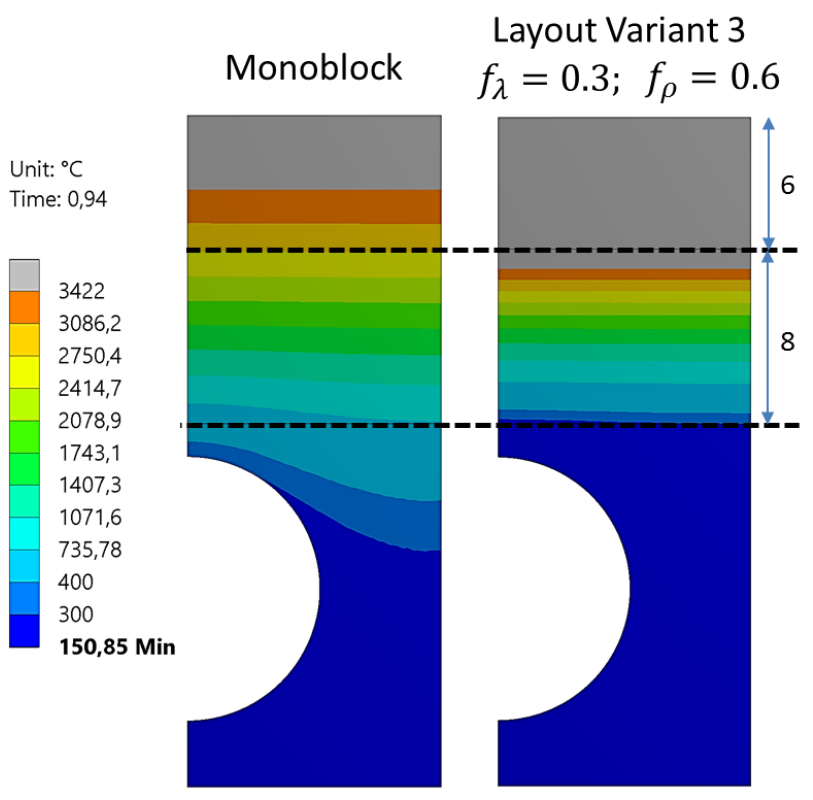

Fig. 4. Comparison variant $3 /$ monoblock under $100 \mathrm{MJ} / \mathrm{m}^{2}$. Armor degradation is expected, but Layout Variant 3 promotes vapor shielding while hindering heat sink overloading
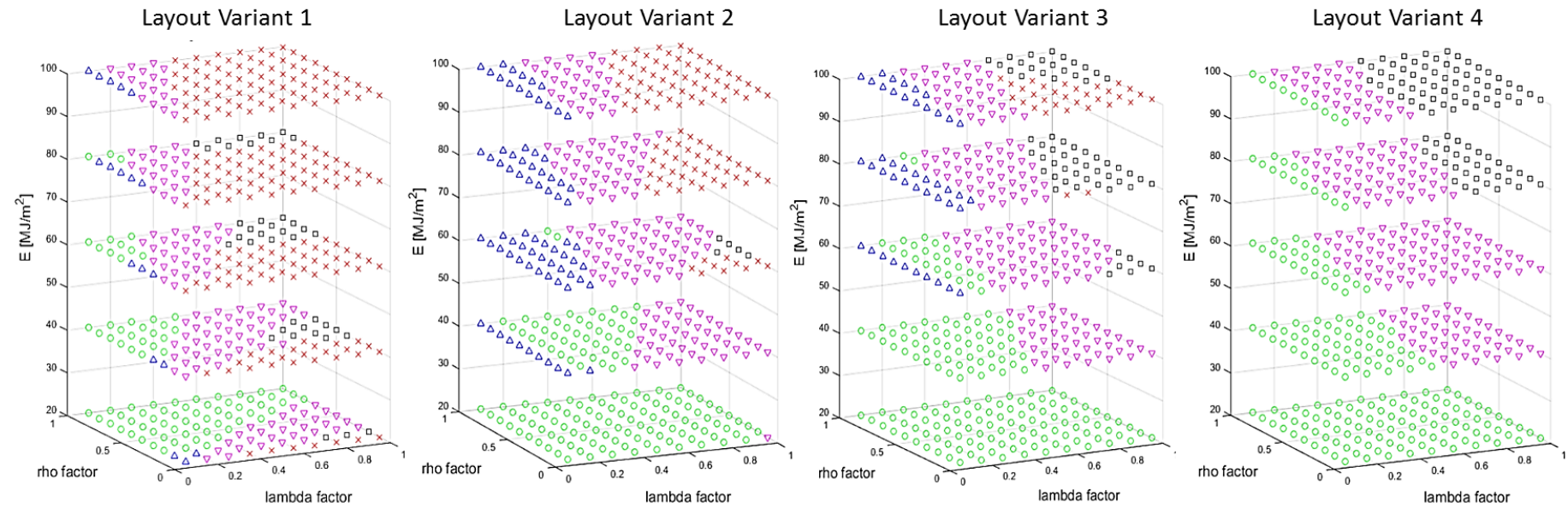

Fig. 5. Parametric study results on the impact of the substrate material and energy deposition (for each layout variant, fully dense W corresponds to the option where both rho and lambda factors are equal to 1) 
Both deposited energy and the substrate's thermal diffusivity turned out to play a decisive role in the global performance of the sacrificial PFC. For each layout in Table 1, the case with only a dense $\mathrm{W}$ armor can be always identified: it corresponds to the combination of the scaling factors equal to 1 for both density ("rho") and thermal conductivity ("lambda"). It appeared that excessive energy deposition due to long pulse duration (tau) and high heat flux magnitude (q) would be challenging handled without occurring in extensive degradation, independently from the chosen layout.

Variant 1 is the case of a $14 \mathrm{~mm}$ thick substrate directly facing the plasma without dense armor. The UJ is set at a distance of $5 \mathrm{~mm}$ from the plasma-facing surface for the qualitative esteem of the thermal gradient through the substrate. In this case, the space of the solutions is narrow. Nonetheless, a number of design points able to withstand consistent energy up to $80 \mathrm{MJ} / \mathrm{m}^{2}$ still exist, confirming the need for further studies in this regard. In Fig.4, the temperature distribution of layout variant 3 is compared with that of a fully dense $\mathrm{W}$ armor, with $100 \mathrm{MJ} / \mathrm{m}^{2}$ deposited in $10 \mathrm{~ms}$. Component degradation is observed in both cases. However, a dense armor on top of a weakly conductive interlayer seems to be a promising option: it offers almost perfect shielding of the heat sink, reducing the risk of overloading and failure. Although vapor shielding was not modelled in our simulation, it should be promoted in such variant, with an almost immediate drop of the impinging heat flux. In this view, the number of events before the replacement or maintenance of the limiter could be increased just by increasing the thickness of the dense sacrificial armor. In Fig.5 the space of solutions is linked directly to the deposited energy $\mathrm{E}$ (product of tau and q). The possibility to cope with transients with significant energy (up to $100 \mathrm{MW} / \mathrm{m}^{2}$ ) can be achieved by means of proper calibration of the substrate material. Better performances are reached by combinations of low conductivity and high density. However, limits imposed by conventional manufacturing routes of $\mathrm{W}$ would impede a flexible combination of density and conductivity. Innovative techniques such as additive manufacturing may provide enhanced design freedom.

\section{Tailored Lattice Structures}

As a result of the parametric study, porous substrates having low thermal conductivity $\lambda$ and high density $\rho$ could perform better than bulk $\mathrm{W}$ during thermal shocks. Their micro-engineered substrate features shall be designed in order to reach a target thermal diffusivity

$$
\alpha=\frac{\lambda}{\rho c_{p}}
$$

As a result, prompt vapor shielding and insulation of the heat sink can be achieved while spreading the energy exhaust over a longer time after the disruption. This imposes also higher steady-state temperatures than bulk $\mathrm{W}$, but the cell and ligament geometry will be such to mitigate significantly the developed thermal stress [18]. As a further step in this direction, a solid model was employed to produce lattice structures tailored to meet the imposed operating criteria. Following the same procedure suggested in [12], thermal conductivity and relative density were evaluated using SolidWorks and ANSYS for several variants of the cell. Among the configurations proposed in Fig.6 for the regular Kelvin cell, the "C Cell" was selected. Its constant ligament cross-section was preferred to achieve high relative density up to $50 \%$ and to address the potential needs of additive manufacturing. The equivalent thermal conductivity at room temperature was always kept below $65 \mathrm{~W} / \mathrm{mK}$, as desired. In Table 3, all the relevant parameters of the model calibration are listed: $\mathrm{L}$ is the ligament length, $\mathrm{r}$ the minimum radius of the ligament cross-section, $\bar{\rho}$ relative density, $\lambda$ thermal conductivity.

It was also observed that both $\bar{\rho}$ and $\lambda$ are not affected by geometrical scaling (factor $\mathrm{k}$ ). This provided us with a flexible parameter to help accommodate potential limits imposed by the manufacturing route, e.g. due to powder size or diameter of the laser beam of additive manufacturing devices. The last two cell variants in Table 3 have been selected as the most promising and respectively called A Model $(\mathrm{A}=1.6)$ and $\mathrm{B}$ Model $(A=0.5)$. An upscaling factor $k$ ranging from 1 to 5.56 was applied based on manufacturing requirements. Ultimately, CAD models were produced consisting of 10x10x10 mm cubes, obtained through linear repetitions of the elementary cell (Fig.7). It is foreseen to exploit additive manufacturing to print samples out of these geometries for material characterization.
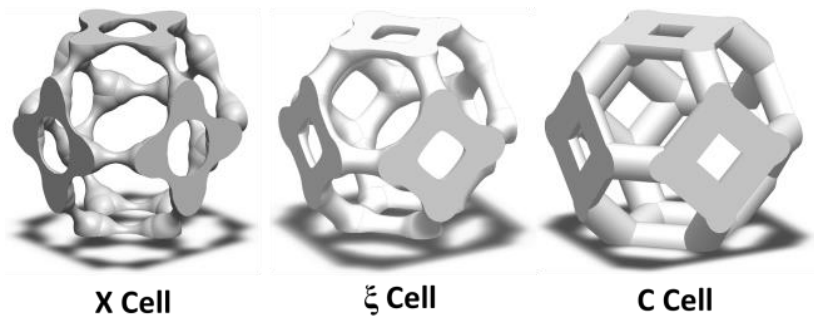

Fig. 6. Three alternative design of the regular Kelvin cell

\begin{tabular}{ccccccc}
\multicolumn{6}{l}{ Table 3. Proposed cell variants and their parameters } \\
$\begin{array}{c}\text { Cell } \\
\text { Type }\end{array}$ & $\mathrm{A}$ & $\begin{array}{c}\mathrm{L} \\
(\mathrm{mm})\end{array}$ & $\begin{array}{c}\mathrm{r} \\
(\mathrm{mm})\end{array}$ & $\begin{array}{c}\bar{\rho} \\
(\%)\end{array}$ & $\begin{array}{c}\lambda \\
(\mathrm{W} / \mathrm{mK})\end{array}$ & $\mathrm{k}$ \\
\hline $\mathrm{X}$ & 0.5 & 0.16 & 0.025 & 18.0 & 7.2 & $\mathrm{~N} / \mathrm{A}$ \\
$\xi$ & 2 & 0.185 & 0.035 & 17.9 & 13.7 & N/A \\
$\mathrm{C}$ & 0.7 & 0.2 & 0.06 & 24.5 & 21.5 & N/A \\
$\mathrm{C}$ & 0.5 & 0.19 & 0.073 & 36.1 & 38.0 & N/A \\
\hline $\mathrm{C}$ & 1.6 & 0.2 & 0.09 & 49.6 & 63.9 & 1 to 5.56 \\
$\mathrm{C}$ & 0.5 & 0.2 & 0.09 & 53.1 & 48.9 & 1 to 5.56 \\
\hline
\end{tabular}

\section{Conclusion}

In the present work, the development of a FEM-based tool to allow parametric optimization of substrate material for a DEMO sacrificial limiter is presented. Layout variants, materials and boundary conditions of this parametric study are described together with the post-processing routine implemented to compare and plot each design point with respect to user-defined criteria. It turned out that both deposited energy and substrate thermal 
diffusivity play a significant role in the global behavior of the PFC during disruptions. Based on these results, innovative lattice structures have been proposed having tailored material features to fulfil imposed functional requirements. Our solid model was employed to design

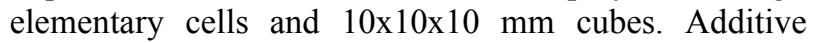
manufacturing techniques will be employed to print out samples for material characterization. Gas trapping tests are also recommended to address licensing requirements related to tritium retention. On the other hand, more detailed information and material characterization are needed. Better esteem of the operating requirements, including thermal shock duration, magnitude and wetted area could be valuable inputs. More detailed FE analyses accounting for melting and vaporization are planned. Ultimately, as mentioned, $\mathrm{W}$ lattices may bring decisive structural benefits. They will be addressed in a dedicated work also including real measurements directly performed on additive manufactured samples. In fact, the structural behavior of lattice structures is expected to be strongly dependent on the geometrical features of the elementary cell itself, so a homogenized approach (where these are neglected) could have led to misleading results.
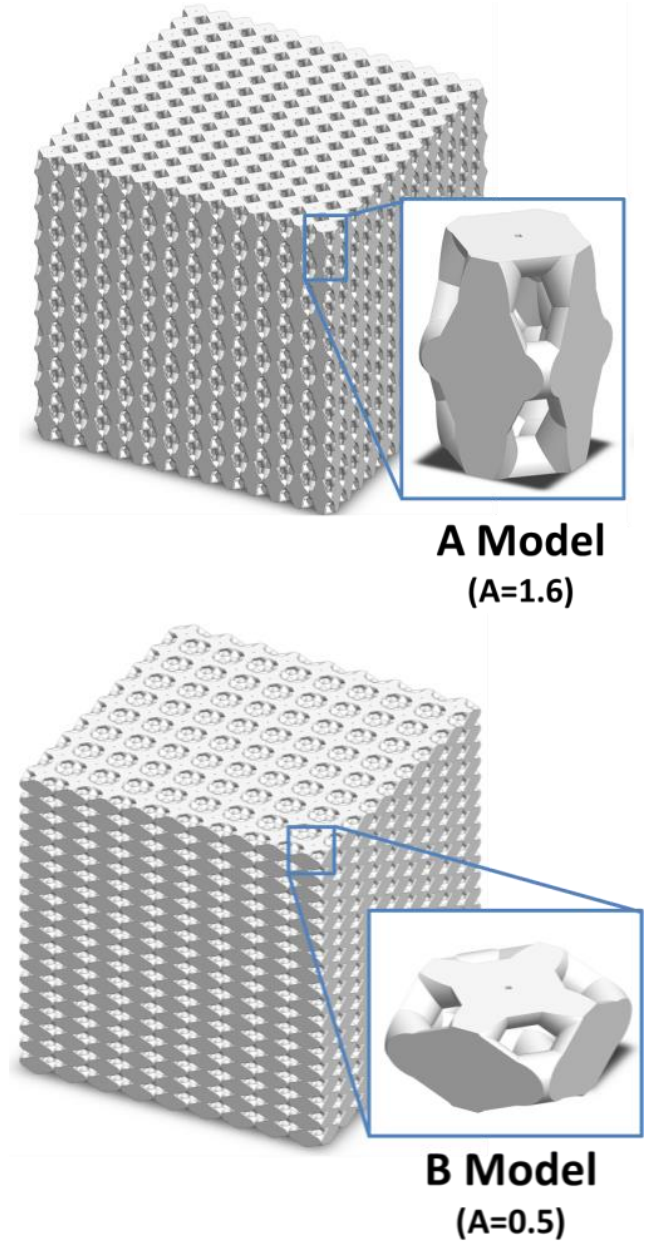

Fig.7. $10 \times 10 \times 10 \mathrm{~mm}$ cubes based on the $\mathrm{C}$ cell type $(\mathrm{k}=1.67)$

\section{Acknowledgements}

This work has been carried out within the framework of the EUROfusion Consortium and has received funding from the Euratom research and training program 2014-2018 and 2019-
2020 under grant agreement No 633053. The views and opinions expressed herein do not necessarily reflect those of the European Commission.

\section{References}

[1] F. A. J. H. Donnè, "The European roadmap towards fusion electricity", Philosophical Transactions of the Royal Society A: Mathematical, Physical and Engineering Sciences, vol. 377, no. 2141, p. 20170432 (2019).

[2] T. R. Barrett, et al. "Progress in the engineering design and assessment of the European DEMO first wall and divertor plasmafacing components", Fusion Engineering and Design 109-111, 917-924, (2016)

[3] F. Maviglia, et al., "Wall protection strategies for DEMO plasma transients", Fusion Engineering and Design, Volume 136, Part A, 410-414 (2018)

[4] R. Wenninger, et al., "The DEMO wall load challenge", Nuclear Fusion 57, 046002(11pp) (2017).

[5] E. Nardon, "Introduction to Disruptions", 9th ITER International School, (2017).

[6] S. Pestchanyi, et al., "Simulation of divertor targets shielding during transients in ITER", Fusion Engineering and Design 109-111, 141145 (2016).

[7] T. R. Barrett, et al., "Designs and technologies for plasma-facing wall protection in EU DEMO", Journal of Nuclear Fusion 59, 056019, (2019).

[8] S. Sharafat, et al., "Micro-engineered first wall tungsten armor for high average power laser fusion energy systems", Nuclear Materials 347, 217- 243 109-111 (2005).

[9] Z. Xiao, et al., "Evaluation of topology-optimized lattice structures manufactured via selective laser melting", Matetial \& Desgin, Volume 143, 5, 27-37 (2018)

[10] A. von Müller, et al., "Additive manufacturing of pure tungsten by means of selective laser beam melting with substrate preheating temperatures up to $1000{ }^{\circ} \mathrm{C}$, Nuclear Materials and Energy 19,184-188, (2019)

[11] P. Fanelli, et al., "Modelling and characterization of structural behaviour of Al open-cell foams", Materials and Design 114, 167175 (2017).

[12] R. de Luca, et al., "Preliminary investigation on $W$ foams as protection strategy for advanced FW PFCs", Fusion Engineering and Design, 10.1016/j.fusengdes.2019.03.017 (2018).

[13] J.H. You, et al. "European divertor target concepts for DEMO: Design rationales and high heat flux performance", Journal of Nuclear Materials and Energy 9, 171-17, (2018)

[14] S. Pestchanyi, et al., "Preliminary results of TOKES simulations for DEMO disruptive divertor targets damage and future plans", KIT conference proceedings, (2017).

[15] ITER Organization, "Historical Baseline Document: Appendix A, Materials Design Limit Data", Approved Version (2013).

[16] E. Rabaglino et al., "Prediction of heat transfer in water actively cooled plasma-facing components", 2000, XVIII Congresso Nazionale sulla Trasmissione del Calore

[17] A.R. Raffray, at al. "Critical heat flux analysis and $R \& D$ for the design of the ITER divertor", Fusion Engineering and Design 45, 377-407, (1999).

[18] W.-Y. Jang, et al., "On the crushing of aluminum open-cell foams: Part I. Experiments" International Journal of Solids and Structures 46, 617-634 (2009) 